\title{
Évolution du système alimentaire haut-alpin
}

Quand l'approvisionnement et la diversité remplacent les productions locales

Evolution of food system in Hautes-Alpes: when supply and diversity replace local productions

\section{Aurélie Rousselot-Pailley}

\section{OpenEdition}

\section{Journals}

Édition électronique

URL : http://journals.openedition.org/ethnoecologie/1009

DOI : 10.4000/ethnoecologie.1009

ISSN : 2267-2419

Éditeur

Laboratoire Eco-anthropologie et Ethnobiologie

Référence électronique

Aurélie Rousselot-Pailley, «Évolution du système alimentaire haut-alpin », Revue d'ethnoécologie [En ligne], 2 | 2012, mis en ligne le 12 novembre 2013, consulté le 01 mai 2019. URL : http:// journals.openedition.org/ethnoecologie/1009; DOI : 10.4000/ethnoecologie.1009

Ce document a été généré automatiquement le 1 mai 2019.

Revue d'ethnoécologie est mis à disposition selon les termes de la licence Creative Commons Attribution - Pas d'Utilisation Commerciale - Pas de Modification 4.0 International. 


\section{Évolution du système alimentaire haut- alpin}

Quand l'approvisionnement et la diversité remplacent les productions locales

Evolution of food system in Hautes-Alpes: when supply and diversity replace local productions

Aurélie Rousselot-Pailley

1 Depuis quelque temps, les pays occidentaux, et d'autres, font face à des crises qui remettent en cause le système économique prévalent (Leuschel 2008). Dans le milieu de la presse comme dans les milieux scientifiques, de plus en plus d'auteurs critiquent à voix haute l'économie de marché, la globalisation des cultures matérielles et immatérielles, les inégalités entre pays pauvres et pays riches, le problème croissant de la faim dans le monde (Ziegler 2003). Une prise de conscience généralisée de la part des citoyens et des politiques voit le jour : il faut sans plus tarder protéger notre environnement et notre patrimoine si nous voulons qu'un jour la majorité de la population bénéficie d'un niveau de vie décent.

2 Avec ce constat qui implique directement notre responsabilité, nous remettons également en question notre agriculture, intensive par définition dans les pays développés. Maintenant que nous n'avons plus l'inquiétude du manque de nourriture, l'agriculture et l'origine de nos aliments redeviennent un sujet d'actualité, au cœur de nos préoccupations quotidiennes comme du temps des pénuries (Ferrières 2006). Mais elle est aussi au cœur des grands enjeux de la planète car ses impacts sur l'environnement et sur la santé humaine sont de mieux en mieux connus (Benachour \& Sralini 2009, Belpomme 2004). Du point de vue alimentaire, cette prise de conscience se traduit au sein d'une partie de la population par une remise en cause de la qualité des produits et du système d'approvisionnement alimentaire. Il existe dorénavant un discours largement admis qui prône un retour vers une agriculture plus respectueuse de l'environnement et des hommes (produits fermiers, produits de terroir, labels de qualité, produits issus de 
l'agriculture biologique, ou issus d'Associations pour le Maintien d'une Agriculture Paysanne).

3 À l'heure de l'émergence du marché mondial et de produits standardisés pour la consommation de masse, l'agriculture de montagne - et qui plus est de haute montagne a dû conserver sa spécificité afin de rester compétitive. Elle est pour le consommateur synonyme d'authenticité et de naturalité. En montagne, les paysages et la biodiversité sont des enjeux importants du patrimoine constituant un écosystème particulièrement précieux mais fragile, notamment parce qu'il est la réserve d'eau douce de la planète, et parce que si la nature à l'état pur n'existe plus depuis longtemps, la montagne en reste tout de même l'un des derniers refuges ${ }^{1}$. Selon la définition du Larousse, le patrimoine est «le bien qu'on tient par héritage de ses ascendants», il est à la fois naturel et culturel, matériel et immatériel ${ }^{2}$. Et c'est en protégeant la biodiversité et en conduisant des politiques de développement durable que nous préserverons le patrimoine, car la nature et les activités anthropiques sont indissociables. Le rythme actuel des transformations économiques et sociales font de la conservation et la transmission des héritages matériels mais aussi immatériels l'objet d'un enjeu mémoriel et identitaire de plus en plus affirmé.

Dans le présent article, nous présentons le cas d'une population montagnarde française chez laquelle nous avons étudié ces questions. Notre étude se situe dans le département des Hautes-Alpes, département intra-alpin et frontalier de l'Italie dans les Alpes du Sud françaises. Nous nous sommes intéressée à la population vivant sur le territoire du Pays des Écrins ${ }^{3}$, dans le Briançonnais, qui se trouve dans la zone périphérique du Parc National des Écrins créé en 1973. Notre objectif est de décrire «la relation à l'alimentation » de la population locale, et d'identifier les marqueurs de changement du système alimentaire local.

5 Le territoire est entièrement rural, et même montagnard ${ }^{4}$. Il resta longtemps enclavé, et la population locale n'a atteint un niveau et des conditions de vie comparables à ceux de la moyenne française qu'au milieu $\mathrm{du} \mathrm{xx}^{\mathrm{e}}$ siècle. Le mode de vie de ces montagnards a profondément et rapidement changé ; ils sont passés du statut de producteur à celui de consommateur, en deux générations, ils ont rattrapé un retard d'un siècle. Leur alimentation, par sa composition et sa place dans l'organisation sociale, s'est radicalement transformée.

6 Aujourd'hui, pris entre deux mondes : celui de la ruralité et celui du modèle urbain, quel sens donnent-ils aux pratiques alimentaires anciennes? Quels sont les modes d'approvisionnement, de consommation et de transmission des savoirs culinaires locaux, qu'il s'agisse de recettes, de productions ou d'exploitation du milieu?

\section{Méthodologie de l'étude}

7 C'est sur l'analyse d'archives communales et départementales, ainsi que sur les données d'une enquête, que nous avons basé notre étude. Cette enquête a été réalisée au cours d'une recherche doctorale menée entre 2005 et 2008. Elle comprend un échantillon de 102 personnes natives de la zone territoriale décrite plus haut, ou installées sur l'une des communes depuis plus de vingt ans. L'étude du corpus d'archives a permis dans un premier temps de comprendre et décrire le contexte socio-économique et alimentaire des Haut-Alpins sur une période allant du début du $\mathrm{xIx}^{\mathrm{e}}$ siècle au début $\mathrm{du} \mathrm{xx}^{\mathrm{e}}$. Ensuite, l'enquête aborde maintes questions relatives à l'alimentation de la population étudiée. De 
cette manière, bien que les données ne soient pas strictement comparables, les connaissances issues des archives et les données qualitatives et quantitatives (entretiens semi dirigés et questionnaires) issues de l'enquête sont complémentaires dans le sens où il en ressort un continuum temporel, du début du xIx ${ }^{e}$ au début du XXI ${ }^{e}$ siècle.

\section{Les Haut-Alpins et leur milieu}

8 Le département des Hautes-Alpes doit son nom au fait que son altitude moyenne est la plus élevée de France, avec de nombreux sommets culminant à plus de 3000 mètres d'altitude et de hautes vallées glaciaires (Chaudet et Pons 1975). L'aridité du relief est sans doute la raison pour laquelle le département a toujours été peu peuplé. Pour une superficie totale de $5549 \mathrm{~km}^{2}$ la population compte aujourd'hui 132000 habitants (chiffres INSEE 2005), soit une densité de 24 habitants $/ \mathrm{km}^{2}$. Le maximum démographique fut atteint en 1846 avec environ 135000 habitants (Brun 1995). Ensuite, et plus particulièrement à la fin $\mathrm{du} \mathrm{xIX}^{\mathrm{e}}$ siècle, le département connaît une pression démographique et foncière qui provoquera l'immigration de nombreux fils et filles $\mathrm{du}$ pays, et donc une diminution rapide de la population, situation qui ne s'inversera qu'après la seconde guerre mondiale (Vidal-Fontille 1988). C'est à partir de cette date que des changements drastiques s'opèrent dans le mode de vie des Haut-Alpins.

Jusqu'à l'ouverture des voies de communication par la construction du chemin de fer à la fin du XIXe siècle et, à partir du début du $\mathrm{Xx}^{\mathrm{e}}$ siècle, par celle de routes carrossables, les Haut-Alpins restèrent relativement enclavés, et plus encore les hautes vallées de la Durance ${ }^{5}$. Cet isolement partiel, qu'il soit dû au positionnement des hameaux ou aux aléas climatiques saisonniers, obligeait les montagnards à ne dépendre que très faiblement des apports extérieurs. Pour survivre dans de telles conditions, il leur fallut apprivoiser leur environnement et miser sur plusieurs types de ressources. Ne comptant que sur luimême, le Haut-Alpin était à la fois agriculteur, éleveur, fromager, boucher, chasseur, mais aussi charpentier, cordonnier, bûcheron, menuisier, vannier. Ces gens étaient des paysans-cultivateurs, et montagnards de surcroît, réunis en communautés villageoises solidaires. Leurs savoir-faire étaient innombrables, leurs connaissances du milieu approfondies. Il faut reconnaître leur mérite : un milieu rude, un travail permanent et harassant entièrement tourné vers la survie, des conditions de vie sans confort... . Ils ont arraché à la montagne une existence sans compromis; pour cela travail et autonomie étaient les maîtres mots. Néanmoins, ce mode de vie obligeait la relation entre hommes et milieu à être l'objet d'un équilibre harmonieux, et ce fut le cas puisque leur gestion du milieu naturel demeura viable pendant des dizaines de siècles. Aujourd'hui, seuls les plus anciens peuvent encore témoigner de ce passé. Nulle part en France, en montagne ou dans les campagnes, ce mode de vie n'a perduré.

Jusqu'à l'après-guerre, le foncier était divisé en d'innombrables petites parcelles où la polyculture dominait et dont l'exploitation était de type familial. Vivant dans les mêmes conditions, la grande majorité de la population avait les mêmes besoins (Mallé 1999) ; tous devaient produire les mêmes denrées agricoles (blé froment, seigle, orge, avoine, légumineuses, pommes de terre, légumes de plein champ...). En parallèle, chaque famille élevait un petit cheptel de bestiaux garantissant un apport en viande et en produits laitiers : une bonne dizaine de brebis, une ou deux vaches et quelques chèvres, un ou deux cochons et de la volaille. Il s'agissait bien d'une économie de subsistance basée sur les productions caractéristiques d'un mode de vie quasiment autarcique à cause de la 
difficulté de procéder à des échanges sur de longues distances du fait du relief. Cependant, cette économie n'était pas totalement fermée car ces montagnards devaient se procurer à l'extérieur les marchandises indispensables qu'ils ne pouvaient produire eux-mêmes, tels les métaux, le cuir, le sel, le café, le sucre, l'huile d'olive, la morue salée, le riz, les épices (Rousselot-Pailley 2008). Pour cela, et une fois prélevé sur la production totale ce dont la famille avait besoin pour l'année à venir, les paysans vendaient le surplus de leurs productions: céréales, laine, brebis et agneaux dans les foires saisonnières; jambons salés, œufs et fromages sur les marchés locaux.

\section{Les ressources agricoles et alimentaires au $\mathrm{XIX}^{\mathrm{e}}$ siècle}

11 Depuis le Moyen Âge, époque caractérisée par les grands défrichages et une période climatique plus chaude que de nos jours (Le Roy Ladurie 1983), les céréales (seigle et blé froment) formaient l'essentiel de l'alimentation des paysans sud-alpins. Les modes de culture céréalières et maraîchères rencontrées dans les Hautes-Alpes étaient les mêmes que pour le reste de la France et de l'Europe établies sur un mode triennal (Mendras 1995). Mais au cours de la seconde moitié du XIX siècle, l'agriculture connut des évolutions. D'une part, le cheptel devint plus important grâce aux prairies artificielles (luzerne, trèfle, sainfoin) et à la culture de plantes fourragères (avoine, orge, betteraves, ers) ${ }^{6}$ qui fournit, en plus du foin, du fourrage aux animaux d'élevage pendant les longs mois d'hiver. En retour, l'augmentation du cheptel accrut considérablement la quantité de fumier disponible et les rendements des cultures. D'autre part, avec l'ouverture des axes de communication, il devint plus facile d'importer du blé de Provence ou du Piémont italien à un moindre coût, ce qui entraîna la diminution des surfaces dédiées aux céréales au profit de nouvelles cultures devenues prioritaires à partir de 1852 (Thivot 1995). Ainsi, la culture de la pomme de terre, plante bien adaptée à l'altitude et dont les rendements élevés protègent la population des disettes, augmenta rapidement jusqu'à ce qu'elle devienne, avec le pain, la base de l'alimentation des populations alpines. À cette époque se développe également la culture des légumes verts que l'on trouve dans les jardins potagers, situés le plus souvent à proximité des habitations. De même, la production des légumes secs (lentilles, fèves, pois, haricots coco blanc et rose) se généralise. Les vergers, eux aussi, sont en nette progression depuis le début du siècle. On y trouve les classiques pommiers, poiriers, cognassiers, pruniers, cerisiers, quelques abricotiers et des amandiers dans le sud du département. Et également, des framboisiers, groseilliers et cassissiers au coin des jardins. On rencontre aussi un grand nombre de noyers dans tout le département. Ce sont des arbres qui résistent bien au climat montagnard, la limite climatique se trouve aux alentours de 1300 mètres d'altitude. Les cerneaux de noix étaient pressés sous une meule de forme spécifique, afin d'en extraire une huile savoureuse. Cette huile constituait la seule matière grasse végétale produite localement en petite quantité et utilisée dans l'alimentation.

12 La richesse essentielle du Dauphiné d'en Haut (Han 1977) sont les montagnes pastorales, et les ovins sont depuis longtemps l'animal privilégié de l'élevage haut-alpin. Depuis l'époque médiévale au moins, de nombreux troupeaux communaux et transhumants, venus de basse Provence (Leveau 2007), envahissent les étages alpins dès le mois de juin. Malgré leur statut d'éleveur, les haut-alpins mangeaient peu de viande en comparaison du reste de la France (Tableau 1). Les paysans préféraient obtenir l'argent de la vente d'une bête plutôt que de la sacrifier pour eux-mêmes. Les familles plus riches qui 
détenaient un troupeau important pouvaient se le permettre plus souvent. Par contre, les populations urbaines consommaient davantage de viande de boucherie, surtout de la viande bovine, les bouchers des villes s'approvisionnant auprès des cultivateurs locaux et en dehors du département pour les bovins. Dans le Queyras cependant, la viande de vache était de consommation courante car les paysans en élevaient beaucoup, les pâturages s'y prêtant davantage (Arnaud 1983).

Tableau 1 : Comparaison de la consommation de viandes - Hautes-Alpes et France

Source : Thivot 1995

13 L'élevage du porc a permis de pallier le manque de viande et a fini par supplanter les autres types de viande consommés auparavant (Prost, 2004). Dès lors, on trouve presque systématiquement dans les familles de cultivateurs deux ou trois cochons dont la viande était consommée soit fraîche, soit salée. La viande salée (poitrine, jambons, épaules, côtelettes) et les morceaux conservés en terrine dans la graisse pouvaient se garder tout au long de l'année. Elle était consommée quotidiennement : on ajoutait un morceau de lard dans la soupe pour le goût ou on en emportait aux champs pour casser la croûte. La graisse de cochon (le saindoux), indispensable à la ménagère, était conservée dans des " toupines $»^{7}$ et servait en cuisine pour les préparations revenues à la poêle. Avec les boyaux et la chair de cochon, on faisait des saucisses de viande ou de chou qui étaient conservées par séchage. La conservation était un problème crucial auparavant. Les viandes non salées étaient cuisinées et mangées juste après la mise à mort de l'animal. Par exemple, dans certains hameaux, pour profiter d'une consommation plus régulière de viande fraîche, les familles d'un quartier tuaient à tour de rôle un agneau, une brebis, une chèvre et se partageaient les morceaux.

Le fromage était aussi une des bases de l'alimentation alpine (Boëtsch \& Ferrié 1999). Dans le Briançonnais, la proportion la plus usuelle consistait à réserver les deux tiers du lait pour le fromage et un tiers pour le beurre afin de produire deux fois plus de fromage que de beurre (Manteyer 1937). Ici, les gens appelaient «tomme » une forme de faisselle qu'ils égouttaient et laissaient vieillir plus ou moins longtemps. Une partie de la production de beurre et de fromage était vendue sur les foires et marchés des environs ou exportée vers les plaines voisines. Jusqu'au milieu du XIx ${ }^{e}$ siècle, la production fromagère se déroulait dans un cadre strictement familial. C'était le travail des femmes et les revenus qu'elles en tiraient leur restaient en propre (Briot 1907). Dans le cadre de la consommation familiale, le beurre subissait une préparation particulière lui procurant un temps de conservation plus long : il était fondu, décanté, mis dans une toupine et conservé au frais dans la pièce ménagère, située dans la partie nord de la maison, ou à la cave.

15 Pour finir, les animaux de basse-cour et les produits carnés issus de la chasse constituaient un complément à l'alimentation quotidienne non négligeable car ces produits carnés étaient consommés frais, ce qui les changeait de la viande salée. Les poules et les poulets, les lapins, les pigeons étaient réservés à la table familiale lors du repas dominical ou pour des repas festifs. Dans le temps, les gens chassaient 12 mois par an. Il existait des réglementations, comme les permis par exemple, mais les chiffres qui suivent montrent bien à quel point ils ne représentaient pas la réalité. Au milieu du XIX siècle, le nombre de permis de chasse délivrés chaque année était faible : 213 en 1845 et 226 en 1852, alors que l'équivalent de huit kilogrammes de poudre par porteur de permis 
étaient vendus annuellement et que le département comptaient environ 1200 chiens de chasse (Thivot 1995). Ce qui signifie que la plupart des paysans chassaient sans permis. Les principaux animaux chassés étaient la perdrix, la caille, le lièvre, le lapin, la grive, le merle, le chamois, le chevreuil, la marmotte et le coq de bruyère. La peau du gibier et le gibier lui-même pouvaient être vendus et rapportaient ainsi un peu d'argent. La marmotte, aujourd'hui dédaignée, et dont la consommation bien réelle pourtant est souvent négligée dans les écrits (Ladoucette 1848), pouvait faire l'objet d'un commerce. Pour cela, le paysan s'en allait en montagne une fois l'automne installé pour plusieurs jours pendant lesquels il déterrait à l'aide d'une pelle des familles entières de marmottes. Il rentrait une fois la mule chargée de ces rongeurs restés léthargiques grâce au froid et entassés dans de grands sacs. Rentré au village, il vendait les marmottes à ceux qui en voulaient. La marmotte constituait une sorte de "gibier à domicile » dans le sens où la famille de marmottes pouvait être enterrée dans un terrier reconstitué dans un coin de la cave. Ainsi, on pouvait ponctionner au fur et à mesure des besoins l'une d'elles pour la cuisiner en civet. Après dégraissement, on la laissait tremper dans l'eau courante pendant 24 heures pour lui ôter son goût de terre.

\section{Du passé millénaire au monde moderne}

16 Avec le désenclavement de la fin du XIX ${ }^{\mathrm{e}}$ siècle, le Briançonnais s'ouvrit encore davantage à l'économie marchande. Et au début du $\mathrm{xx}^{\mathrm{e}}$ siècle, l'ouverture d'usines hydro-électriques créa un précédent : il devint possible pour les gens de la terre de gagner un salaire, ce qui signifiait pour eux avoir un revenu garanti et suffisant pour se procurer à l'extérieur de la stricte production familiale ce dont ils avaient besoin pour vivre. C'était le début de la monétarisation pour ces régions encore très paysannes. Cette époque est aussi marquée par le développement d'un tourisme axé sur le thermalisme et l'alpinisme (Guichonnet 1980). La population se met alors progressivement à développer des structures d'accueil pour les touristes de passage qui représentaient un apport d'argent non négligeable. Depuis les années 70, le tourisme est devenu, avec la construction et le bâtiment, le moteur économique du département. Aujourd'hui, le tourisme emploie $50 \%$ de la population active.

17 Ces éléments de progrès ont définitivement modifié le mode de vie et les coutumes des vallées de la Haute-Durance. Les gens nés avant la seconde guerre mondiale ont grandi dans un contexte où régnait une autarcie partielle supportée par un système agropastoral extensif et un mode d'exploitation familial des terres. La génération suivante, c'est-à-dire leurs enfants, a rompu avec la société communautaire séculaire qui prévalait et l'héritage parental jusqu'alors inévitable. Poussés par la volonté de vivre mieux et souvent ailleurs, nombre d'entre eux ont migré dans un bon « pays ", plus méridional, ou dans les grandes villes de France (Marseille, Grenoble, Lyon, Paris). Leur époque fut celle de l'essor de ce que nous connaissons bien maintenant: la société de consommation. En deux générations, le mode de vie ancestral et l'agriculture de montagne ont disparu. L'alimentation de ces populations a bien évidemment changé de la même manière (Tableau 2).

18 L'abondance alimentaire caractéristique de nos pays nous offre un large choix de produits de qualité, toujours disponibles et correspondant aux valeurs de notre temps : sécurité et diversité. Nos parents, et nos grands-parents surtout, n'avaient accès qu'à des aliments saisonniers en résonance avec leur environnement, leur alimentation; en comparaison 
des composants de notre alimentation quotidienne, était monotone par les préparations culinaires et répétitive par les cultures, ô combien conditionnées par le terrain et le climat. Ils consommaient beaucoup de céréales non raffinées, principalement sous forme de pain, de tourtes, de pâtes façonnées, les soupes étaient faites de légumineuses, de légumes d'automne (choux, navets, poireaux, courges, oignons), d'herbes sauvages et d'aromates dès le printemps, de légumes verts en été, de pommes de terre. Les animaux leur apportaient des œufs et des produits laitiers (lait, beurre et fromages), un peu de viande, du miel (le sucre non naturel était un luxe). Le saindoux et le lard servaient à graisser et donner du goût aux préparations culinaires, avec parfois de l'huile d'arachide, de noix ou d'olive. Ils buvaient du café mélangé ou non à la chicorée, et du vin léger coupé à l'eau. Néanmoins, ce régime répondant fort bien aux recommandations nutritionnelles actuelles du gouvernement français ${ }^{8}$, on peut considérer que leur alimentation était saine d'un point de vue nutritionnel, et sans considérer les périodes de disette 9 .

Tableau 2 : Économie et mode de vie

Au début des années 80 déjà, le paysage des hautes vallées duranciennes avait perdu les marques de la vie paysanne. Aujourd'hui, les champs ne sont plus fauchés ou cultivés mais laissés en friche ou gagnés par les broussailles; les écuries réaménagées en appartement ou en cave. Les petites épiceries de village ont fermé, les foires biannuelles ont souvent cessé d'avoir lieu, ou tout au moins la part des négoces d'animaux et des grains a fortement diminué ou est inexistante. En revanche, des magasins d'alimentation de plus en plus grands ont ouvert leurs portes et accueilli dans leurs nombreux rayons abondamment achalandés des gens heureux de pouvoir enfin goûter au "progrès", comme «ceux d'en bas ».

\section{Lieux d'approvisionnement et pratiques d'aujourd'hui}

Les grandes surfaces sont de loin le lieu d'approvisionnement le plus fréquenté par la population actuelle car ces magasins sont pratiques et économiques. En général, les gens $\mathrm{y}$ font les courses principales, car ils y trouvent tous les produits alimentaires ou non dont ils ont besoin. Mais lorsque leurs activités le leur permettent, ils fréquentent aussi les marchés pour y acheter essentiellement fruits, légumes et fromages, car disent-ils, « ils sont meilleurs que dans les supermarchés». Les marchés des alentours (Vallouise, l'Argentière, Briançon) sont très appréciés pour la qualité et le choix des produits proposés, et les femmes en particulier reconnaissent volontiers l'aspect convivial de ce moment " où l'on rencontre du monde, où l'on papote ».

Une partie du questionnaire abordait la question de l'offre alimentaire dans ses aspects positifs et négatifs. De façon presque unanime, les consommateurs considèrent le système agro-alimentaire sécurisé et relativement transparent. Cependant, dans le discours général, on perçoit une certaine méfiance vis-à-vis des produits vendus en supermarché car "on ne sait ni comment ils sont faits ni ce qu'il y a dedans». Et nombre de produits chimiques et "non identifiables » entrant dans la chaîne alimentaire, du champ à l'assiette, sont souvent montrés du doigt par les plus jeunes, mais considérés comme un mal nécessaire par les plus âgés. Finalement, les critiques négatives concernent plutôt la grande distribution et portent essentiellement sur les aspects de la qualité et de la fraîcheur des denrées périssables, et parfois sur le grand nombre de produits proposés 
qui inspire un sentiment de surabondance inutile. Avec cette remarque, est souvent évoquée la notion de goût, de parfum et de saveur des aliments qui, de ce point de vue, déçoivent un bon nombre d'acheteurs en grande surface. Enfin, les produits achetés directement aux producteurs sont très valorisés par les consommateurs: ils sont considérés de bonne qualité, parce que "l'on sait d'où ça vient et comment c'est fait », et puis, quand on achète les produits en grande quantité, le prix de revient est intéressant. Il n'est pas rare en effet que les gens achètent auprès d'un éleveur local un agneau entier, ou une demi-vache à l'automne, dont les morceaux découpés seront congelés pour l'année à venir. Les personnes rencontrées qui critiquent la grande distribution mais qui y font pourtant leurs courses alimentaires justifient leur démarche par le fait qu'elles estiment ne pas avoir vraiment le choix. Que ce soit parce que «la qualité coûte cher», ou parce qu'il n'existe pas d'autres réseaux d'approvisionnement, ou pour ce qui est de l'agriculture biologique, parce qu'elles estiment que cela est un "business " comme un autre dont le but est de faire plus d'argent en coûtant plus cher. Certaines personnes de la catégorie des plus âgées, et qui ont donc souvent elles-mêmes travaillé la terre, se méfient de ce label car pour elles « la terre, l'eau et l'air n'ont pas de frontières ... ». Des « boutiques bio » se sont ouvertes en ville (Briançon) ou dans le bourg de l'Argentière-La Bessée, mais sont absentes des vallées. Nous n'avons rencontré que très peu de personnes $(2 \%)$ qui se nourrissent exclusivement de produits biologiques, car "il faut en avoir les moyens». Nombreux sont les jeunes (de moins de 40 ans) en revanche qui aimeraient pouvoir se le permettre, mais ils trouvent cela trop cher. Cependant, certains font le choix de s'approvisionner pour une partie seulement en « bio », ceux que Claire Lamine appelle les «mangeurs bio intermittents» (2004). Ils y achètent les produits de base (céréales, graines, fruits, légumes, œufs, lait) car "leur coût n'est pas beaucoup plus élevé » que celui des produits issus de l'agriculture conventionnelle.

À côté de cela, les boulangers ont toujours une clientèle bien affirmée. Rares sont ceux qui n'achètent pas leur pain en boulangerie, ou auprès du camion du boulanger quand il passe dans le hameau. Parfois, si la boulangerie est trop loin du village ou que le pain " ambulant » ne leur convient pas, ils le font eux-mêmes. Dans ce cas, beaucoup utilisent une machine à pain, appareil qui s'est fortement répandu dans les foyers campagnards depuis trois ans, mais quelques-uns le font cuire dans un four à pain particulier, construit à cet effet à l'extérieur de la maison. Ils font alors des fournées d'une vingtaine ou plus de pains qu'ils congèlent ensuite.

L'approvisionnement classique dans les magasins d'alimentation est bien souvent complété, quoique cela ne représente qu'une petite partie des besoins annuels, par l'autoproduction. Les gens du "pays", installés dans des maisons familiales possèdent presque toujours de petites parcelles héritées des ancêtres qu'ils peuvent cultiver et parfois des vergers productifs s'ils ont été entretenus correctement. Nombreux aussi sont ceux qui s'autorisent l'élevage de quelques animaux de basse-cour. Dans les potagers, on trouve généralement : pommes de terre, carottes, oignons, échalotes, poireaux, salades, radis, céleris, betteraves rouges, blettes (ou poirée), potimarrons, courgettes, courges, épinards, haricots verts, choux, tomates, persil, basilic, framboises, cassis, groseilles, fraises, rhubarbe. Et parfois aussi, brocolis, concombres, petits pois, haricots cocos, asperges, romarin, sauge, verveine, thym.... Dans les vergers, on trouve essentiellement cognassiers, pommiers, poiriers, pruniers et cerisiers. Quelques-uns se risquent à planter un pêcher ou un abricotier, qui peuvent donner des fruits selon l'exposition. Mais dans le Briançonnais, où les communes s'étagent entre 900 et 1600 mètres d'altitude, la fréquence 
des gels tardifs et la fraîcheur des nuits sont peu favorables à ce type d'arboriculture ${ }^{10}$. Dans les petits élevages des particuliers, on trouve essentiellement des volailles (poules, poulets, canards, dindes, pintades, pigeons), et des lapins, mais ces derniers sont moins courants. Seuls les exploitants agricoles actifs possèdent des bovins, des moutons ou des chèvres. Ils sont alors souvent de grands consommateurs de viande, et profitent de leur statut pour tuer une bête ou deux pour leur propre consommation. Parfois, ils élèvent également, pour eux-mêmes ou pour un partage en famille, un ou deux cochons. Élever un cochon est contraignant, c'est pourquoi c'est devenu rare, même parmi les agriculteurs. D'abord, il faut être là tous les jours, avoir un enclos où le loger, il faut lui donner chaque jour sa nourriture, composée si possible de céréales, de grains et de déchets végétaux. Et enfin, il faut disposer du matériel nécessaire à son sacrifice qui a lieu en général après la première chute de neige, car on tue le cochon quand il commence à faire froid, entre novembre et janvier selon les localités.

Entre les produits du jardin et ceux provenant des producteurs locaux ou régionaux, il est d'absolue nécessité d'avoir les moyens de stocker et conserver cette quantité de nourriture disponible sur une courte période. Pour pouvoir profiter pleinement des productions « maison ", plusieurs modes de conservation existent, ils varient en fonction du type de denrées. Le stockage des denrées alimentaires peut se faire dans une chambre ménagère, en cave ou dans la grange, pratique typiquement rurale (Corbeau 1998), mais aussi grâce aux moyens récents de la stérilisation et de la congélation. Ce qui détermine le mode est la durée de conservation et les propriétés gustatives attendues au moment de la consommation. Le mode de conservation le plus répandu est la congélation. Les femmes l'ont définitivement adopté dès les années 70. Il est vrai que la congélation est un moyen de conservation extrêmement économique en espace de rangement, en temps nécessaire au conditionnement, outre qu'elle garantit une conservation longue et sécurisée des aliments. Par ailleurs, la cave reste un espace très utilisé par ceux qui habitent les maisons traditionnelles. On y trouve en général pommes de terre, carottes, poireaux, betteraves, choux, salades d'hiver, pommes, poires. Cela peut demander un conditionnement, par exemple les placer sur un système de claies ou les repiquer dans de la terre ou du sable apporté à cet effet dans la cave. La technique de la stérilisation pour faire des conserves de fruits et de légumes est encore répandue. Presque toutes les personnes rencontrées déclarent au moins faire quelques confitures, mais certaines font encore des bocaux de légumes et de fruits.

Toutes les étapes qui relèvent de la conservation sont sous la responsabilité des femmes. L'homme quant à lui est traditionnellement chargé de saler les jambons et les épaules du cochon, ou du gibier comme le chamois. Mais la préparation des terrines et des conserves de charcuterie est la tâche de la femme. La saucisse de chou, qui est une des rares spécialités culinaires locales, est fabriquée par les deux partenaires du couple car c'est un travail assez long et fastidieux, mais le séchage des saucisses reste du ressort de l'homme. En ce qui concerne la préparation des apéritifs et des vins, cela dépend davantage du talent de l'un ou du goût de faire de l'autre. Cependant, on peut tout de même relever une préférence selon le sexe : les vins de fruits sont en général confectionnés par les femmes alors que le génépi l'est par l'homme ${ }^{11}$, les plantes sauvages médicinales et culinaires, cueillies à certaines périodes de l'année et séchées, font essentiellement partie du savoir féminin (Delcour 2004).

26 La consommation de gibier reste l'apanage des campagnes. Dans près de la moitié des foyers, l'un des hommes est chasseur et procure à la cuisine familiale de la viande de 
gibier (sanglier, lièvre, chevreuil, chamois, perdrix). Sinon, c'est un oncle ou un ami de la famille qui en donne un peu selon ses prises annuelles. Ces mets sont consommés quand " il y a du monde à la maison", de la famille ou des amis. Ce doit être une occasion un peu spéciale qui justifie de cuisiner ce type de viande car elle symbolise le sauvage, la nature, le goût de ce qui est rare et $a$ fortiori bon. Elle est généralement cuisinée en civet et parfois rôtie, selon le morceau.

\section{Conclusion}

Notre alimentation est le lien qui nous relie à la nature car la production de nos aliments est liée à la terre, à l'eau, au soleil. Elle est le reflet des modes de vie en relation avec les contraintes géo-climatiques, les territoires et les terroirs (Escallier 1989). La manière de « faire les aliments » étant, en quelque sorte, l'expression d'une relation dynamique entre la physiologie des individus et leurs ressources naturelles, leur appartenance sociale, leur culture et leurs représentations. L'alimentation, objet du quotidien, est un « fait total social » (Fischler 1990), et en cela, un prisme de lecture de l'évolution des sociétés.

Nous avons vu qu'à l'échelle du Briançonnais, et similairement pour les Hautes-Alpes, les habitudes alimentaires ont changé parallèlement aux évolutions du contexte socioéconomique. Les habitants d'aujourd'hui connaissent et utilisent encore les ressources locales qu'offre la montagne, même si cela est beaucoup moins généralisé qu'autrefois. Les pratiques alimentaires, que ce soit de l'ordre de la production de denrées ou des préparations culinaires, s'inscrivent souvent dans un registre d'usages anciens et considérés par les gens eux-mêmes comme traditionnels. Les motivations à l'origine de la pérennité de ces pratiques sont associées à l'aspect économique d'une part, et ludique d'autre part. Par exemple, l'auto-production est la garantie d'une certaine autonomie spatiale et économique car on dispose à volonté de produits naturels et de saison entrant dans l'alimentation quotidienne. Cela représente également une économie de temps en épargnant à la cuisinière de parcourir des kilomètres en voiture pour acheter la salade de midi au magasin ou au supermarché le plus proche. Ludique, parce que pour les hommes et les femmes de cette région, l'exploitation des ressources faunistiques et floristiques locales, mais aussi le fait de cultiver un potager ou de faire son bois pour l'hiver, est l'objet d'une passion ou d'un passe-temps, d'un savoir-faire et de connaissances héritées des « anciens ». Cette recherche nous a permis d'identifier des continuités et des ruptures dans les pratiques et les habitudes alimentaires de la population étudiée. La modernité a réduit l'impact du milieu sur les hommes, et changé celle de l'homme sur le milieu qu'il exploite différemment, mais elle n'a pas rendu complètement inutiles certaines pratiques traditionnelles. Si certaines pratiques ancestrales sont devenues obsolètes et ont disparu alors que le contexte socio-économique changeait, d'autres, en revanche, ont survécu. L'alimentation est sans aucun doute le résultat et l'expression du mode de vie, mais elle est aussi et surtout constitutive d'une tradition léguée par les anciens qui fournit le substrat nécessaire à la construction identitaire d'aujourd'hui. Car ce qui est traditionnel est vivant et ne cesse de se recréer pour mieux s'adapter à travers les gestes mis en œuvre.

Par ailleurs, depuis une vingtaine d'années, on assiste à une "rurbanisation» des campagnes et de la montagne du fait du développement des résidences secondaires, du tourisme et de l'urbanisation touristique, du retour des retraités dans leur région d'origine. Espaces et paysages sont donc à partager entre agriculteurs, néo-ruraux et 
touristes, ce qui ne va pas toujours sans difficulté car chacun a son usage de la montagne. Comme l'écrit Jean-Pierre Poulain (2007), urbains et rurbains voient les espaces ruraux de plus en plus souvent de façon paysagée, romantisée, qui ne laisse guère de place à la fonction et même aux traditions agricoles. Cependant, nous avons observé de la part des néo-ruraux, qui représentent près de la moitié de la population permanente, mais aussi des jeunes natifs du pays, une réelle volonté de renouer avec les activités agricoles et certains aspects culturels d'autrefois. L'héritage des pratiques alimentaires passe ainsi par un processus multiple de transmission: vertical entre les générations, horizontal entre les groupes sociaux.

Cette transmission participe également à la revalorisation de la culture montagnarde car il est vrai que le travail des agriculteurs est primordial pour l'entretien et la préservation des paysages et de leur diversité dans leur aspect fonctionnel. Les hommes, qui ont façonné pour répondre à leurs besoins ces paysages tant appréciés aujourd'hui, étaient des paysans-cultivateurs, ils «cultivaient» le "pays». Ce que certains appellent les " nouvelles fonctionnalités de l'agriculture » (Bassignana 2006), ne sont pas si nouvelles que cela. Les systèmes de polyculture-élevage, par leur complémentarité et la nécessité d'économie à tous les niveaux de production du système alimentaire, étaient des modèles de recyclage. Les vertus de la multifonctionnalité de l'agriculture que l'on redécouvre consistent à faire ce que les paysans-cultivateurs faisaient déjà, c'est-à-dire du développement durable avant l'heure.

31 Si nos paysages et toutes les espèces végétales et animales qui y contribuent sont considérés comme un patrimoine naturel, notre gastronomie et nos habitudes alimentaires doivent l'être également, mais en termes de patrimoine immatériel. Ce concept, créé en 2003 par l'Organisation des Nations Unies pour l'éducation, la science et la culture, consacre le " patrimoine culturel vivant ", comme étant le véritable creuset de la diversité culturelle et le garant du développement durable. Le concept de développement durable est le résultat d'une évolution importante des idées, de la notion de "protection» de la nature, qui opposait l'homme et ses activités, à la notion de «conservation». Il admet dès lors un usage raisonné des espaces à protéger et intègre conjointement les enjeux de la biodiversité et de l'activité humaine. Or, la montagne est un milieu naturel qui accueille une diversité culturelle phénoménale (Greenwood 2008), notamment parce que la difficulté des échanges et des communications d'une vallée à l'autre, et les conditions géo-climatiques variant d'un versant à l'autre, ont favorisé le développement de populations différentes sur des échelles géographiques réduites. D'autre part, les pratiques d'élevage et de culture en montagne sont très spécifiques au milieu. La force des contraintes naturelles nécessite le respect de cycles saisonniers, et la fragilité de l'équilibre établi entre les ressources et les activités humaines oblige les différents usagers de la montagne à travailler en concertation ${ }^{12}$. 


\section{BIBLIOGRAPHIE}

Arnaud C. 1983 - Une mémoire de Saint-Véran, vie traditionnelle et patois queyrassins. Le monde alpin et rhodanien $\mathrm{n}^{\circ} 3$.

Bassignana M. 2006 - Préface à l'ouvrage d'André Bornard, Les végétations d'alpage de la Vanoise. Description agro-écologique et gestion pastorale. Versailles, Quae.

Belpomme D. 2004 - Ces maladies créées par l'homme. Comment La dégradation de l'environnement met en péril notre santé. Paris, Albin Michel. (Essai).

Benachour N. \& Sralini G.-E. 2009 - Glyphosate Formulations Induce Apoptosis and Necrosis in Human Umbilical, Embryonic, and Placental Cells. Chem. Res. Toxicol., 2009, 22(1) : 97-105.

Boëtsch G. \& Ferrié J-N. 1999 - Introduction à l'ethnocuisine alpine. Évolutions biologiques et culturelles en milieu alpin, Actes de l'Université d'été 1999, Éditions CDDP des Hautes-Alpes.

Briot F. 1907 - Les Alpes françaises. Nouvelles études sur l'économie alpestre. Diverses questions générales et monographies. Paris, Berger-Levrault.

Brun J-P. 1995 - Paroisses et communes de France, Dictionnaire d'histoire administrative et démographique - Hautes-Alpes. Paris, CNRS.

Chaudet P. \& Pons P. 1975 - Les Hautes-Alpes, hier, aujourd'hui, demain. Tome premier : La Nature, L'Homme, Gap, Société d'Études des Hautes-Alpes.

Corbeau J-P. 1998 - Comportements alimentaires chez la personne âgée. Objectif nutrition 39. Paris, Institut Danone.

Delcour D. 2004 - Plantes et gens des Hauts, Usage et raison de la flore populaire médicinale hautalpine. Forcalquier, Haute-Provence, Les Alpes de lumière. (Les cahiers de Salagon 9).

Escallier Chr., Robert J-F. \& Desjardins G. 1989 - Inventaire du patrimoine culinaire des Alpes du Sud. Rapport final. CETTAL (Centre d'Études des Techniques Traditionnelles Alimentaires).

Ferrières M. 2006 - Histoire des peurs alimentaires. Du Moyen Age à l'aube du xx ${ }^{e}$ siècle. Paris, Le Seuil. (Points Histoire).

Fischler Cl. 1990 - L'homnivore. Paris, Odile Jacob.

Greenwood G. 2008 - Moutains Regions : Laboratories for Adaptation. International Human Dimensions Programme on global environmental change IHDP UPDATE). October 2008, Issue 2.

Guichonnet P. 1980 - Histoire et civilisations des Alpes, II. Destin Humain. Toulouse, Privat, Lausanne, Payot.

Han A. 1977 - La Vallouise par un de ses fils. La Vallouise touristique. Gap, Louis-Jean.

Ladoucette, baron J. C.-F. 1848 - Histoire, topographie, antiquités, usages, dialectes des Hautes Alpes. Paris, Gide et Cie.

Lamine Cl. 2004 - L'analyse des choix et pratiques alimentaires des « mangeurs bio intermittents », une contribution à une sociologie de la variabilité des pratiques. Texte présenté au Congrès de l'AISLF, Tours, Juillet 2004.

Le Roy Ladurie E. 1983 - Le climat depuis l'an mil. Paris, Flammarion. 
Leuschel R. 2008 - La crise va durer dix ans. Le Soir. 14 octobre.

Leveau Ph. 2007 - Archéologie et géoarchéologie d'une période historique dans les Alpes : les paradigmes épistémologiques de la romanisation. Géomorphologie : relief, processus, environnement, $1: 85-98$.

Mallé M-P. 1999 - L'habitat du nord des Hautes-Alpes, patrimoine architectural et mobilier. Association pour le patrimoine de Provence et la Société d'Études des Hautes-Alpes.

Manteyer G. de 1937 - L'usage du lard et du beurre en Briançonnais (Oulx 1542). Bulletin de la Société d'Études des Hautes-Alpes.

Mendras H. 1995 - Les sociétés paysannes. Éléments pour une théorie de la paysannerie. Paris, Gallimard. (Folio/Histoire). (1éd. 1976, Armand Colin).

Pogneaux N. 2006 - Les paysans de la Haute-Durance $\mathrm{du} \mathrm{xv}^{\mathrm{e}}$ au xix ${ }^{\mathrm{e}}$ siècle. Travaux agricoles et conditions de vie. Cahiers du Château Saint-Jean, 3 : 3-37. Du Fournel, L'Argentière-La Bessée.

Poulain J.-P. 2007 - La relation homme/animal à l'épreuve des modèles alimentaires, L'Homme, Le Mangeur, l'Animal. Qui nourrit l'autre? Les Cahiers de l'Ocha ${ }^{\circ} 12$.

Prost M. 2004 - Éléments et pratiques de l'alimentation carnée dans les Alpes Dauphinoises (xive ${ }_{-}$ xix $^{\mathrm{e}}$ siècles). In Aubaile F., Bernard M. \& Pasquet P. (Dir.) - La viande, un aliment, des symboles. Aix en Provence, Édisud : 103-122. (Écologie Humaine)

Rousselot-Pailley A. 2008 - Les Hauts Alpins au xix ${ }^{\mathrm{e}}$ siècle : mode de vie et alimentation. Cahier du Château Saint-Jean, $\mathrm{N}^{\circ} 6$. L'Argentière-La Bessée, Du Fournel, 90 p.

Thivot H. 1995 - La vie privée dans les Hautes-Alpes vers le milieu du xix ${ }^{e}$ siècle. Gap, Louis-Jean.

Vidal-Fontille Ch. 1988 - La population des Alpes du Sud de 1860 à nos jours. Tome 1. Gap, Éditions Orphys.

Ziegler J. 2003 - Le droit à l'alimentation. Paris, Mille et Une Nuits.

\section{NOTES}

1. OCHA, Le mangeur, la montagne (http://www.lemangeur-montagne.com).

2. Convention pour la sauvegarde du patrimoine culturel immatériel, issue de la Conférence générale de l'UNESCO, réunie à Paris du vingt-neuf septembre au dix-sept octobre 2003 en sa $32^{\mathrm{e}}$ session.

3. Ce territoire compte environ 6000 habitants et comprend neuf communes sur une superficie de $463 \mathrm{~km}^{2}$ : La Roche-de-Rame ; Saint-Martin-de-Queyrières ; L'Argentière-la-Bessée ; Champcella ; Freissinières; Les Vigneaux; Puy-Saint-Vincent; Vallouise; Pelvoux. Toutes les communes situées en rive droite de la Durance ont une partie de leur territoire située en zone centrale du Parc, où l'activité humaine est fortement réglementée.

4. Le territoire étudié est classé en zone montagne, et même haute montagne, car les communes sont situées en partie à plus de 1200 mètres d'altitude et présentent un dénivelé de plus de 400 mètres. Les zones de haute montagne représentent $4 \%$ du territoire français.

5. Rivière importante qui traverse le département du nord au sud et qui forme un axe de communication essentiel depuis l'Antiquité jusqu'à nos jours.

6. Sorte de lentilles pour le bétail.

7. Mot patois désignant une jarre en céramique de petite taille.

8. Deuxième Programme National Nutrition Santé 2006-2010. Actions et Mesures. Ministère de la Santé et des Solidarités. 
9. La dernière datant de la fin du XIX ${ }^{\mathrm{e}}$ siècle pour la région étudiée (Pogneaux 2006).

10. La commune de la Roche de Rame constitue une exception à cette règle. Au début du xx siècle, alors que la Vallouise exportait des pommes jusqu'à Marseille, les habitants de cette commune qui bénéficie d'un microclimat favorable (présence de genévrier thurifère, courant en Afrique du Nord), cultivaient des abricots tardifs à grande échelle pour l'époque et les exportaient.

11. Le génépi est un apéritif commercialisé, mais que la plupart des gens font eux-mêmes dans cette région car la plante est indigène (Artemisia glacialis et Artemisia umbelliformis sont les plus courantes). Elle est très valorisée pour son aspect sauvage car elle pousse entre 2500 et 3500 mètres d'altitude, son goût et ses vertus médicinales.

12. La nouvelle charte du Parc National des Écrins illustre parfaitement cette réalité.

\section{RÉSUMÉS}

La population montagnarde vivant sur le territoire du Pays des Écrins (département des HautesAlpes, France), aujourd'hui très hétérogène, présente un mode de vie qui s’inscrit dans la modernité mais véhiculant toujours des habitudes et des pratiques alimentaires dont on retrouve les origines dans l'histoire de ces sociétés paysannes. La connaissance et l'analyse du milieu et de l'organisation socio-économique et familiale permettent une lecture assez précise des stratégies de transmission des habitudes alimentaires, d'approvisionnement et d'exploitation du milieu naturel environnant.

Dans le cadre des recherches menées au cours de ma thèse de doctorat, une centaine d'entretiens semi-dirigés ont été conduits avec des sujets âgés de 20 ans et plus, permettant une approche intergénérationnelle des pratiques alimentaires. Dans un premier temps, le croisement des données issues de l'étude d'un corpus d'archives et des entretiens menés avec les " anciens » a permis de décrire les contextes environnemental, agricole et alimentaire de la population locale depuis le début du XIX ${ }^{\mathrm{e}}$ siècle, et ainsi d'identifier les marqueurs de transformation des pratiques alimentaires (économie de subsistance versus économie touristique; auto-production versus consommation de produits extérieurs, etc.).

Ensuite, notre intérêt s'est porté sur l'évolution des stratégies d'approvisionnement encore marquées par des spécificités liées à l'environnement montagneux (cueillette, chasse, produits du jardin, du verger, de l'élevage, plats typiques, des modes de conservation). Ces sont d'ailleurs ces spécificités, revendiquées comme marqueurs de l'identité locale, qui font l'objet d'une transmission entre générations et entre groupes sociaux (locaux versus néo-ruraux). Pour finir, nous verrons comment certaines pratiques survivent et s'intègrent à une alimentation rythmée entre autre par les saisons que le milieu rend si prégnantes.

The mountain population living on the territory of the "Pays des Écrins " (department of Hautes-Alpes, France), today very heterogeneous, present a way of life which satys within modernity but still transporting ancient foodways that we can identify in the history of these rural societies. Knowledge and analysis of middle, socioeconomic and family organization allow us to definite the strategies of transmission of food habits, supply and working of the natural environment.

During my research, a hundred of semi-aimed discussions were driven with subjects of 20 and more years old, allowing an "inter-generation» approach of food practices. At first, the 
crossroads of the existent data of the study of a corpus of archives and discussions led with «ancient " allowed us to represent the environmental, agrarian and food contexts of the local population since the beginning of the 19th century. And thus, to identify the markers of transformation of food practices (economy of subsistence versus tourist economy; autoproduction versus consumption of outside products, etc).

Then, our interest turned on the evolution of strategies of supply still marked by peculiarities linked to hilly environment (picking, hunt, gardening, the orchard, animal husbandry, typical dishes, modes of conservation). This are besides these peculiarities, claimed as markers of local identity, which make the object of a transmission between generations and between social groups. To finish, we will see how certain practices survive and integrate with a feeding regulated by the seasons, so determinating in this kind of environment.

INDEX

Keywords : food practices, rural societies, supply, mountain environment, seasons, vertical and horizontal transmissions, sustainable development

Mots-clés : sociétés paysannes, pratiques alimentaires, approvisionnement, environnement montagnard, saisonnalité, transmissions verticale et horizontale, développement durable

\section{AUTEUR}

\section{AURÉLIE ROUSSELOT-PAILLEY}

Le Mas des Bruns

05310 La Roche de Rame

France

a.rousselotpailley@gmail.com 